\title{
Overexpression of EPS8L3 promotes cell proliferation by inhibiting the transactivity of FOXO1 in HCC
}

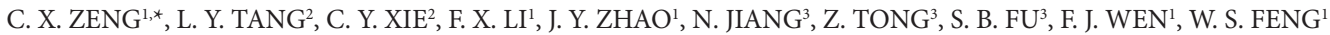

${ }^{1}$ Department of General Surgery, Guangdong Second Provincial General Hospital, Guangzhou, China; ${ }^{2}$ Department of General Surgery, Zengcheng People's Hospital, (BoJi-Affiliated Hospital of Sun Yat-Sen University), Zengcheng, China; ${ }^{3}$ Department of Hepatic Surgery, The Third Affiliated Hospital of Sun Yat-sen University, Sun Yat-sen University, Guangzhou, China

*Correspondence: zxcq12333@163.com

Received July 25, 2017 / Accepted February 1, 2018

\begin{abstract}
The homology of epidermal growth factor receptor pathway substrate 8 (EPS8), EPS8L3, is elevated and significantly enhanced in hepatocellular carcinoma (HCC) tissues and cell lines compared to normal liver tissues and cell lines. MTT and colony formation assays demonstrated that EPS8L3 over-expression induces HCC cell proliferation and silencing reduces it. Further experiments illustrated that over-expressing EPS8L3 promotes p-AKT and Cyclin D1 expression, but inhibits the transcriptional activity of FOXO1. Colony formation assay also demonstrated that AKT inhibitor suppresses the effect of EPS8L3 on proliferation in EPS8L3-over-expressing cells, while AKT restores the proliferation of EPS8L3-silenced cells. This suggests that EPS8L3 promotes proliferation by hyper-activating the AKT signaling pathway and subsequently inhibiting FOXO1 transcriptional activity. Our results provide a new view on EPS8L3 and human HCC progression and therefore EPS8L3 may prove a novel therapeutic target for HCC.
\end{abstract}

Key words: hepatocellular carcinoma, EPS8L3, FOXO1, signaling pathway

Liver cancer is the sixth most frequent incidence cancer and the second leading cause of cancer related death. China alone accounts for approximate $50 \%$ of the entire number of liver cancer cases and deaths [1,2]. More than $90 \%$ liver cancer is hepatocellular carcinoma (HCC) [3] and the prognosis is poor because of high metastasis and relapse rate, so it is imperative to understand the molecular mechanisms involved and find effective HCC therapy.

One of the most important HCC characteristics is rapid proliferation, and this contributes to cancer progression. Recent knowledge in oncogenes and signaling pathways that modulate cancer cell proliferation has increased, and here the epidermal growth factor receptor pathway substrate 8 (EPS8) particularly sparked our interest. EPS8 was initially discovered by the expression cloning approach [4] and later studies demonstrated that it is located in human chromosome 12 p13.2 and is universally expressed $[5,6]$. Further research showed that EPS8 is over-expressed in various human solid tumors and hematology malignancy, and it also included the following; (1) Chu et al. illustrated that EPS8 was over-expressed in oral squamous cell carcinoma and its deregulation closely related with poor survival
[7]; (2) Gorsic et al. showed that over-expression of EPS8 elevated resistance to cisplatin, and that knockdown EPS8 increased sensitivity to cisplatin in lung cancer cells [8]: (3) Chen et al's. immunohistochemical assay found that EPS8 was up-regulated in $>60 \%$ of human breast cancer and that this up-regulation enhanced the capability of cell growth, migration and invasion in breast cancer [9]; (4) studies proved that EPS8 enhanced cell proliferation by regulating cascade responses such as the PI3K/AKT/mTOR, Raf/Mer/ Erk and STAT pathways [9] and (5) there are at least three other EPS8-like proteins in mammals with similar amino acids and structure. These are EPS8L1, EPS8L2 and EPS8L3. $[5,10-12]$.

Although the precise mechanism of EPS8L3 in HCC remains unclear, we found it is significantly elevated in HCC tissues and cell lines. While EPS8L3 over-expression markedly promotes HCC cell line proliferation, down-expression inhibits it, and further study has shown that EPS8L3 can down-regulate FOXO1 transactivity by activating the AKT signaling pathway. Our results demonstrate that EPS8L3 functions as an oncoprotein in HCC progression and that it can therefore provide a novel therapeutic target in HCC. 


\section{Materials and methods}

Tissue specimens. All tissues were collected between 2012 and 2014 at Zengcheng District People's Hospital (BoJi-Affiliated Hospital of Sun Yat-Sen University). Prior informed patient consent and approval from the Institutional Research Ethics Committee were obtained for use or the study specimens, and all experiments were performed in accordance with all relevant guidelines and regulations.

Cell lines. Mortalized human liver epithelial cells (Nor-1, Nor-2 and Nor-3) and HCC cell lines (HepG2, Huh7, Hep3B, QGY-7703, BEL-7402, MHCC97H and MHCC97L) were cultured in Dulbecco's Modified Eagle's Medium (DMEM; Gibco, Grand Island, NY, USA) and 10\% fetal bovine serum was added (FBS; HyClone, Logan, UT, USA). All cell lines were maintained at $37^{\circ} \mathrm{C}$ in a humidified incubator under $5 \% \mathrm{CO}_{2}$.

Vectors and retroviral infection. Over-expressed EPS8L3 plasmid was constructed by sub-cloning the full-length human EPS8L3 cDNA into pMSCV. Two human siRNA sequences targeting EPS8L3 were cloned into pSuper-retropuro for the down-down of EPS8L3 with the following siRNA sequences: RNA\#1 CCTAAAGCTATCAACCTGCTACTCGAGTAGCAGGTTGAT AGCTTTAGG, RNA\#2 CCAGAAGATCAAGCACAGCTTCTCGAG AAGCTGTGCTTGATCTTCTGG. The reporter plasmid for quantitatively examining the transcriptional activity of FOXO1 was constructed in the pGL3-Enhancer plasmid (Promega, Madison, Wisconsin, USA) [13]. Transfection was performed with Lipofectamine 3000 reagent (Invitrogen, Carlsbad, CA, USA) and stable cell lines were selected for 10 days with $0.5 \mu \mathrm{g} / \mathrm{mL}$ puromycin.

RNA extraction and real-time quantitative polymerase chain reaction (RT-qPCR). Total RNA was extracted with Trizol reagent (Invitrogen, Carlsbad, CA, USA) according to instruction and cDNAs were amplified in the ABI 7500 Fast System (Applied Biosystems, Rockville, MD, USA). The housekeeping GADPH gene was used as reference and the relative expression of mRNA was calculated by the algorithm $2^{-[(\mathrm{Ct} \text { of gene)-(Ct of GAPDH)], }}$, where $\mathrm{Ct}$ represents the threshold cycle.

The primers were: EPS8L3, forward 5'-AGCCATTTACTTGCACCGGAA- 3 ' and reverse 5'-CTCCCCTGCTTGCATGTCAT-3'; Cyclin D1, forward 5'-CAATGACCCCGCACGATTTC-3' and reverse 5'-CATGGAGGGCG GATTGGAA-3'; GAPDH forward: 5-ACCACAGTCCATGCCATCAC-3' and reverse: 5-TCCACCACCCTGTTGCTGTA-3'.

Western blotting assay. Cells were lysed with RIPA buffer [50 mM Tris-HCl (pH 7.2), $150 \mathrm{mM} \mathrm{NaCl}, 1 \%$ Triton $\mathrm{X}-100,1 \%$ sodium deoxycholate, $0.1 \%$ SDS and cocktail protease inhibitors]. Then $30 \mu \mathrm{g}$ lysate was heated at $98^{\circ} \mathrm{C}$ for 10 minutes and separated on 12\% SDS-PAGE gel and the protein was transferred to PVDF membrane. The membrane was probed with primary antibody against EPS8L3, p-AKT, AKT, p-FOXO1, FOXO1 Cyclin D1 and $\alpha$-tubulin (diluted
1:3000, Sigma-Aldrich, St Louis, MO, USA) at $4^{\circ} \mathrm{C}$ overnight. The membrane was then incubated by HRP-conjugated secondary antibody (diluted 1:2500, Sigma-Aldrich, St Louis, MO, USA) and $\alpha$-tubulin was used as the loading control.

MTT assay. Cells in $3 \times 10^{3}$ volume were seeded in 96-well plates and dye-stained for 4 hours with $0.5 \mathrm{mg} / \mathrm{mL}$ MTT (3-(4,5-Dimethyl-2-thiazolyl)-2, 5-diphenyl-2H-tetrazolium bromide - Sigma, St Louis, MO, USA). Cell absorbance was measured at $490 \mathrm{~nm}$ wavelength.

Colony formation assay. The $5 \times 10^{2}$ cell volume was seeded in 6-well plates and incubated for 14 days, and colonies were then fixed in 10\% formaldehyde and dyed with $1 \%$ crystal violet for 30 seconds.

Luciferase activity assay. $5 \times 10^{4}$ cells were implanted in 24 -well plates for 24 hours, and $100 \mathrm{ng}$ luciferase reporter plasmids and $10 \mathrm{ng}$ pRL-TK renilla plasmid were then transfected into the indicated cell with Lipofectamine 3000 (Invitrogen, Carlsbad, CA, USA). Luciferase and renilla activity was detected 48 hours later.

Microarray data analysis. Microarray data was downloaded from Cancer Genome Atlas (TCGA; http:// cancergenome.nih.gov) and extracts were performed by $\mathrm{MeV}$ v. 4.6 software (http://www.tm4.org).

Statistical analysis. All experiments were repeated three times and statistical analysis was performed by SPSS 19.0. Data is presented as mean $\pm \mathrm{SD}$, with $\mathrm{p}<0.05$ statistically significant.

\section{Results}

EPS8L3 is over-expressed in HCC compared to normal liver and adjacent non-tumor and cirrhotic tissues. Results from The Cancer Genome Atlas datasets (TCGA) analysis illustrated that relative EPS8L3 mRNA expression is significantly increased in HCC tissues compared to normal liver tissues (Normal: $n=50$, HCC: $n=351 ; \mathrm{p}<0.001$; Figure 1A). The HCC tissues also expressed more EPS8L3 than the corresponding adjacent non-tumor tissues (ANT; $n=50 ; p<0.001$; Figure 1B). Further analysis of EPS8L3 expression in different liver diseases also provided relatively elevated mRNA expression compared to normal liver tissues (normal), adjacent non-tumor tissues (ANT) and cirrhotic to HCC tissues. The expression decreased in that order (Figure 1C).

RT-qPCR then assayed EPS8L3 expression in fresh normal liver tissues, cirrhotic tissues and HCC tissues and the results indicated that EPS8L3 expression is highest in HCC tissues and lowest in normal liver tissues (Figure 2A). Western blotting and RT-PCR assays provided markedly increased EPS8L3 expression in paired normal liver tissues and HCC tissues compared to the matched adjacent non-tumor tissues (ANT; Figure 2B and C). Finally, we checked our results in the human immortalized normal liver epithelial cells (Nor-1, Nor-2 and Nor-3) and HCC cell lines (HepG2, Huh7, Hep3B, QGY-7703, BEL-7402, MHCC97H and MHCC97L) .We established that EPS8L3 expression was sharply elevated 
A

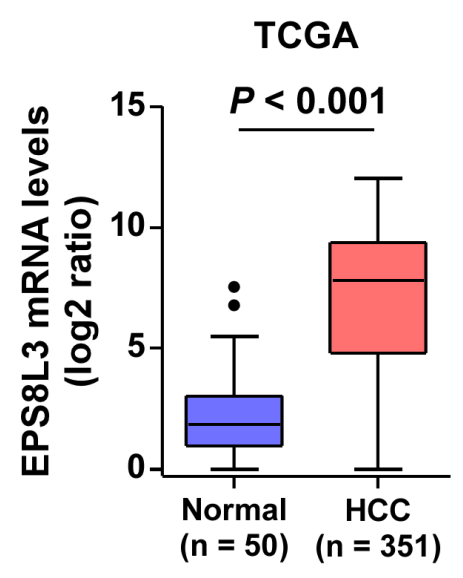

B

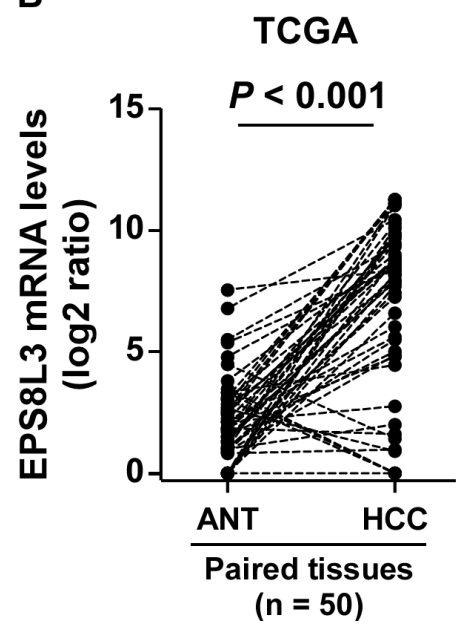

C

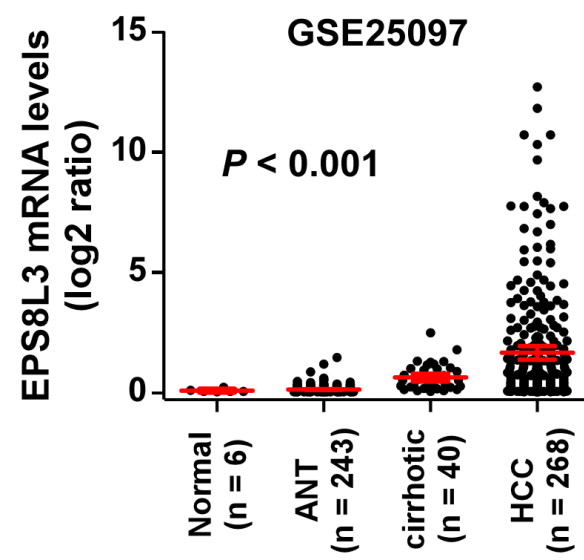

Figure 1. The expression profile of EPS8L3 in HCC. A) The mRNA levels of EPS8L3 in normal liver tissues $(n=50)$ and HCC tissues $(n=351)(p<0.001$; TCGA). B) The mRNA level of EPS8L3 in 50 paired adjacent non-tumor tissues (ANT) and HCC tissues (Tumor). C) The mRNA levels of EPS8L3 in normal liver tissues (normal, $n=6$ ), adjacent non-tumor tissues (ANT, $n=243)$, cirrhotic $(n=40)$ and HCC tissues $(n=268)(p<0.001$; GSE25097).

A

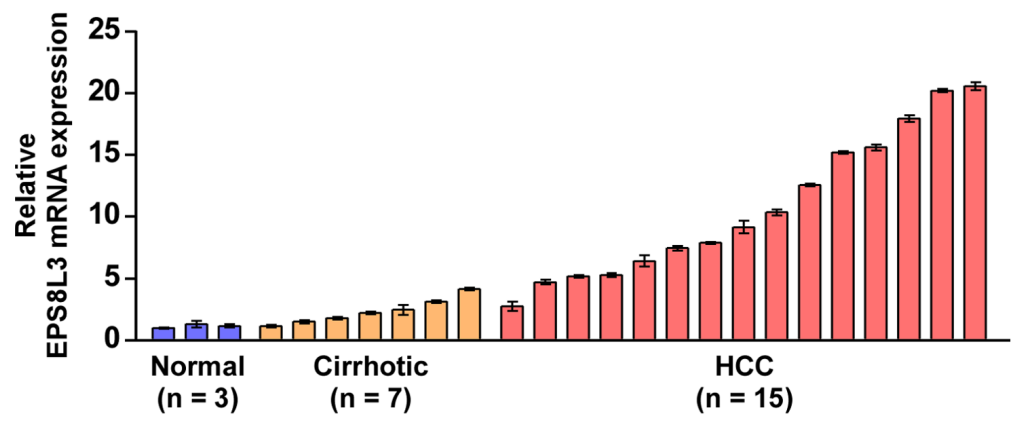

C
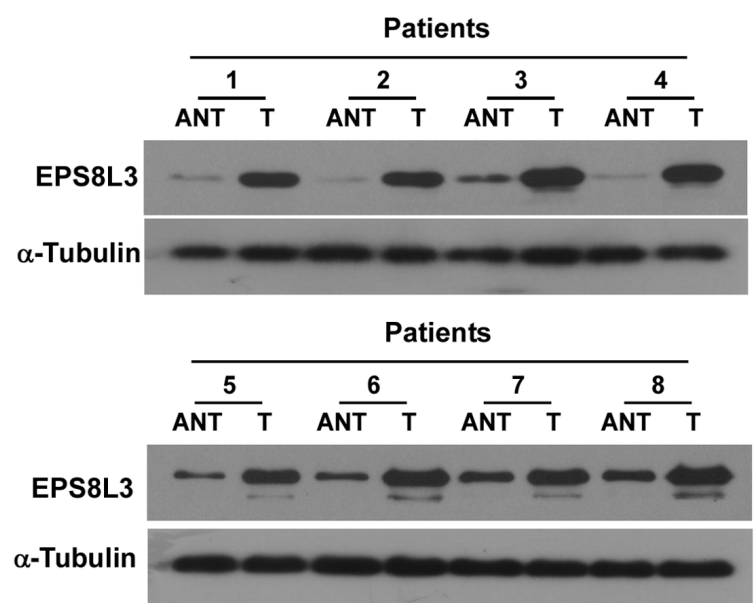

B

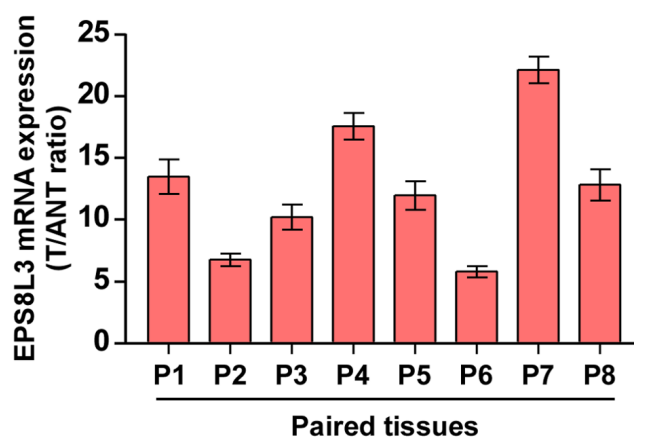

D

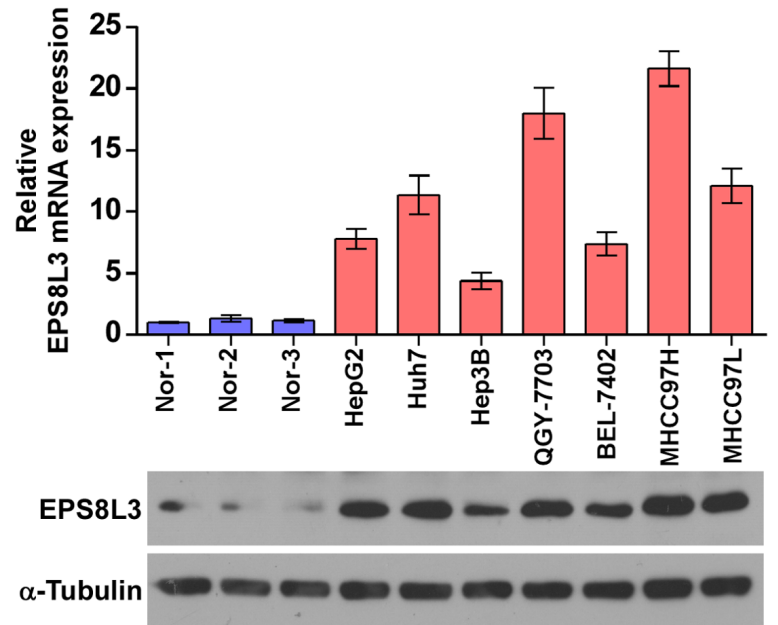

Figure 2. The expression of EPS8L3 in HCC tissues and cell lines. A) The relative mRNA expression of EPS8L3 in fresh normal liver tissues (normal, $n=3)$, cirrhotic tissues $(n=7)$ and HCC tissues $(n=15)$. B) The mRNA expression ratio of HCC tissues $(T)$ to corresponding adjacent non-tumor tissues (ANT). C) The protein levels of HCC tissues (T) and corresponding adjacent non-tumor tissues (ANT). $a$-tubulin was used as the loading control. D) The relative mRNA expression (upper panel) and protein levels (lower panel) of EPS8L3 in the human immortalized normal liver epithelial cells (Nor-1, Nor-2 and Nor-3) and HCC cell lines (HepG2, Huh7, Hep3B, QGY-7703, BEL-7402, MHCC97H and MHCC97L). $\alpha$-tubulin serves here as the loading control. 
in HCC cell lines compared to immortalized normal liver epithelial cells at both transcription and translation levels (Figure 2D). Therefore, EPS8L3 was significantly overexpressed in HCC tissues and cell lines.

Upregulation of EPS8L3 enhances the proliferation of the HCC cell line. The impact of EPS8L3 on proliferation of HCC cells was evaluated by gain- and loss-of-function models. We over-expressed EPS8L3 in Hep3B with low EPS8L3 level and inhibited it in MHCC97H with a high level (Figure 3A). The MTT assay showed that the proliferation rate of up-regulated cell EPS8L3 is significantly higher than that in corresponding vector cells, but EPS8L3 silencing drastically inhibited this cell proliferation (Figure 3B). We further confirmed results with colony formation assay and,
A

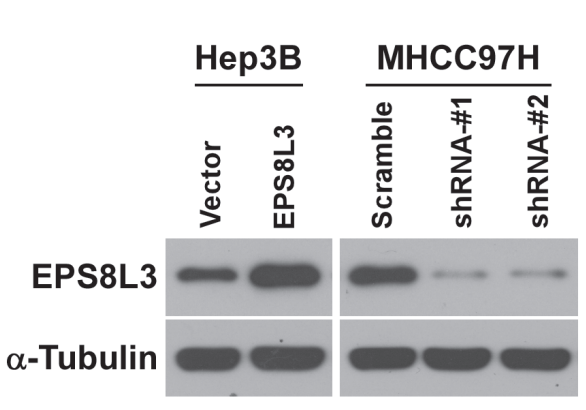

C

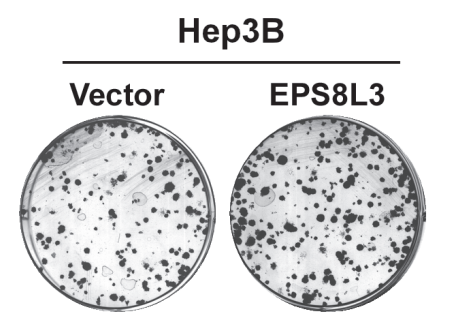

B

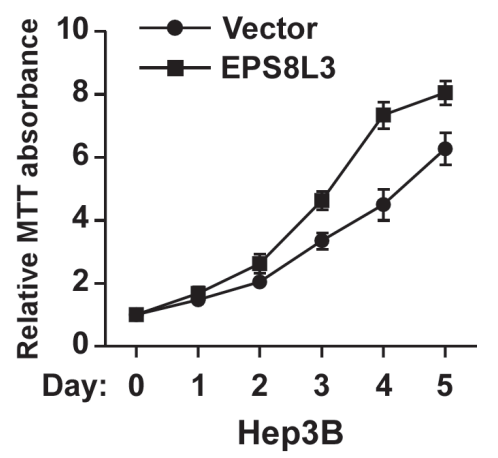

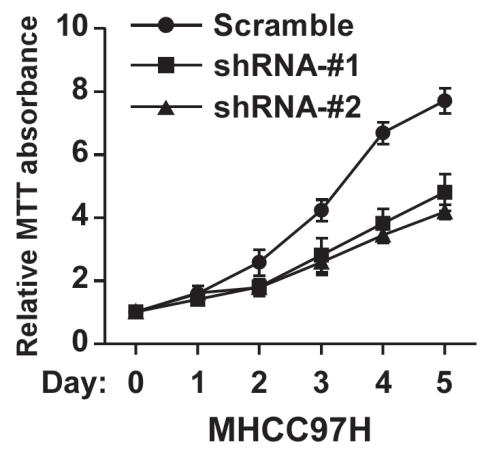

Нер3в
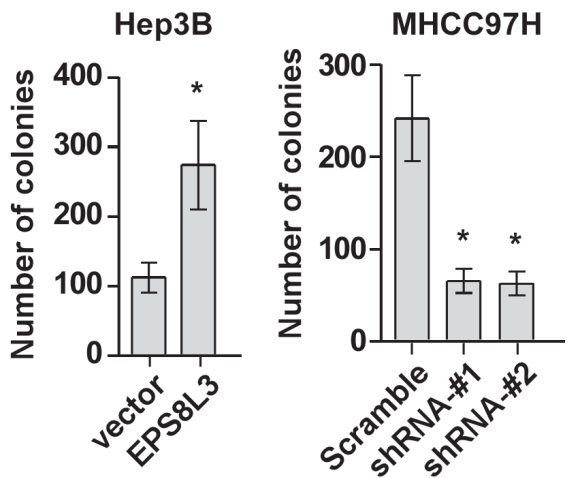

MHCC97H

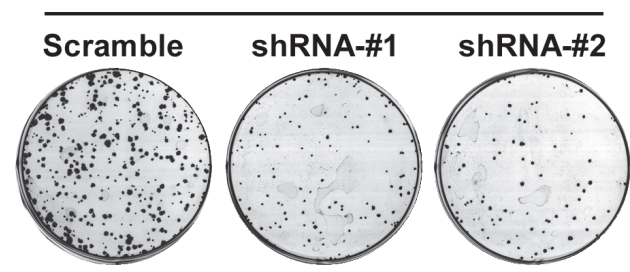

Figure 3. Overexpressing EPS8L3 promoted proliferation of HCC cells. A) The EPS8L3 protein expression in gain- and loss-function cell models, with a-tubulin as the loading control. B) The relative proliferation rate of EPS8L3-overexpressing cells (left panel) and EPS8L3-silencing cells (right panel) by MTT assay. C) The representative images (left panel) and colony numbers (right panel) of colony formation assay in EPS8L3-overexpressing cells and EPS8L3-silencing cells.

A

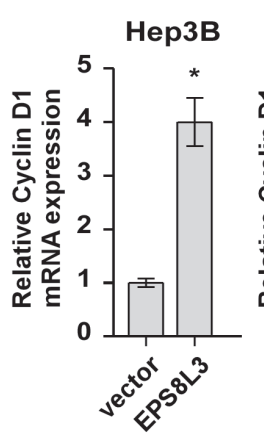

MHCC97H

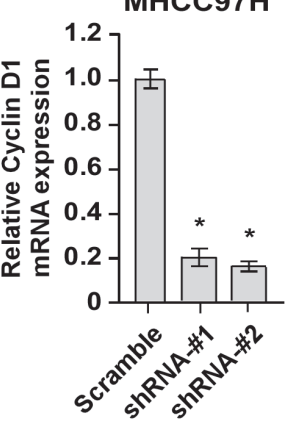

B

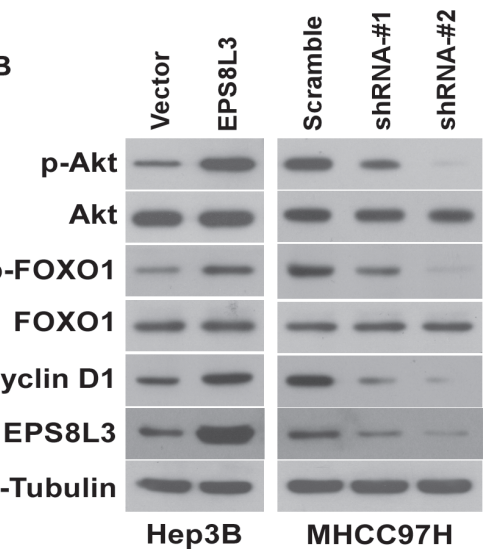

C

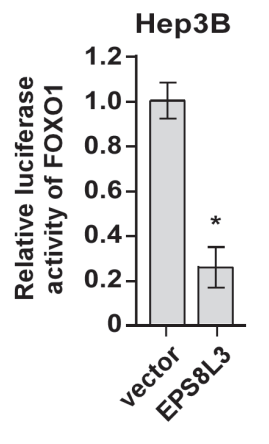

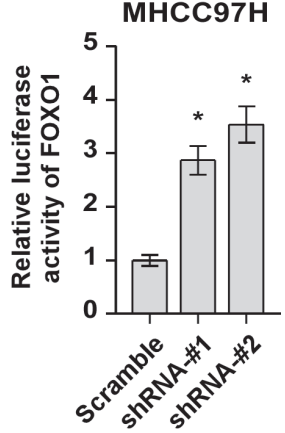

Figure 4. EPS8L3 inhibits FOXO1 transactivity and activates the AKT pathway. A) The relative Cyclin D1 mRNA expression in EPS8L3-over-expressing cells and EPS8L3-silencing cells. B) The protein expression of p-AKT, AKT, p-FOXO1, FOXO1, Cyclin D1 and EPS8L3 in EPS8L3-over-expressing cells and EPS8L3-silencing cells, with a-tubulin as the loading control. C) The relative luciferase activity of FOXO1 in EPS8L3-silencing cells. 
as in the MTT assay, over-expressed EPS8L3 enhanced colony formation in both the size and number of HCC cells, and silencing reduced it (Figure 3C). These combined results provided strong evidence that EPS8L3 has a pivotal role in HCC cell proliferation.

EPS8L3 inhibits FOXO1 transactivity and activates AKT signaling pathway. Previous studies demonstrated that FOXO1 transcriptionally regulates cell proliferation [14, 15] and this inspired us to determine if EPS8L3 regulates cell proliferation in this way. RT-qPCR and western blotting assays registered drastically increased Cyclin D1 expression in EPS8L3-overexpressed cells and reduced expression in EPS8L3-silenced cells (Figures 4A and B). FOXO1 transactivity, however, produced the opposite trend (Figure 4C). AKT kinase has been reported to play an important role in phosphorylating FOXO1 and weakening its transcriptional activity [16], and while up-regulated EPS8L3 significantly promoted p-AKT expression, suppressed EPS8L3 reduced it, (Figure 4B).This suggests that EPS8L3 enhances cell proliferation via the $\mathrm{AKT} / \mathrm{FOXO1}$ signaling pathway.

Furthermore, colony formation assay demonstrated that although the Perifosine AKT inhibitor, $(20 \mu \mathrm{M}$; Selleck, Houston, Texas, USA), strongly reduces AKT phosphorylation levels (Figure 5A) and suppresses the elevated effect of EPS8L3 on cell proliferation (Figure 5B), AKT restores proliferation of EPS8L3-silenced cells (Figure 5C). These combined results show that EPS8L3 enhances cell proliferation by activating the AKT signaling pathway which inhibits FOXO1 transactivity. Moreover, AKT phosphorylation is essential for HCC progression.

There is positive correlation between EPS8L3 and p-AKT and Cyclin D1. Correlation examination of EPS8L3, p-AKT and Cyclin D1 protein levels in 11 HCC tissues
(Figure 6A) revealed positive correlation between EPS8L3 and Cyclin D1 $(\mathrm{r}=0.47, \mathrm{p}<0.05)$ and $\mathrm{p}-\mathrm{AKT}(\mathrm{r}=0.71, \mathrm{p}<0.05)$ (Figure 6B).

\section{Discussion}

Herein, we established that EPS8L3 was significantly elevated in HCC tissues and cell lines and that its ectopic expression enhanced HCC cell proliferation. The underlying molecular mechanisms include hyper-activation of the AKT signaling pathway and subsequent inhibition of FOXO1 transcriptional activity. These alterations change the expression of genes involved in the cell cycle. Our results therefore provide a new view on EPS8L3 function in the progression of human HCC and they promote EPS8L3 as a novel HCC therapeutic target.

We found that over-expressing EPS8L3 enhanced HCC cell proliferation by stimulating AKT and FOXO1 phosphorylation. This resulted in cytoplasm localization of FOXO1 and loss of cell cycle inhibition. FOXO1 has been reported to be a tumor suppressor gene and it is decreased in multiple cancers; including chronic myeloid leukemia and bladder, endometrial and breast cancers [17-20]. FOXO1 also has an important role in multiple signaling by transcriptionally modulating downstream genes. An example is that FOXO1 inhibits cell proliferation by transcriptional induction of $\mathrm{p} 21^{\mathrm{Cip} 1}, \mathrm{p} 27^{\mathrm{Kip} 1}$ and $\mathrm{p} 57^{\mathrm{Kip} 2}$ which are cyclin-dependent kinase inhibitors (CDK).

Phosphorylated FOXO1 transfers to the cytoplasm and combines with 14-3-3 chaperone proteins, thus preventing transcriptional induction of the above CDK inhibitors [20, 21]. FOXO1 also inhibits Cyclin D1 expression through crosstalk with other transcriptional regulators or by inducing
A

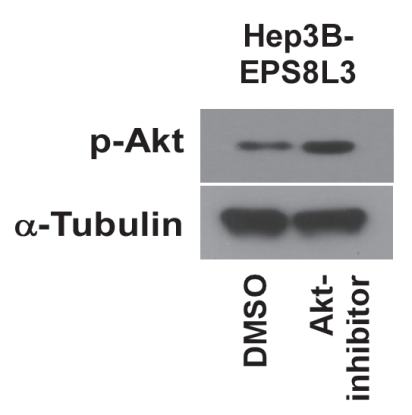

B

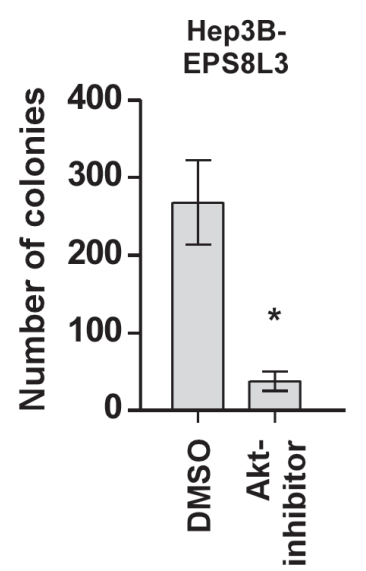

C

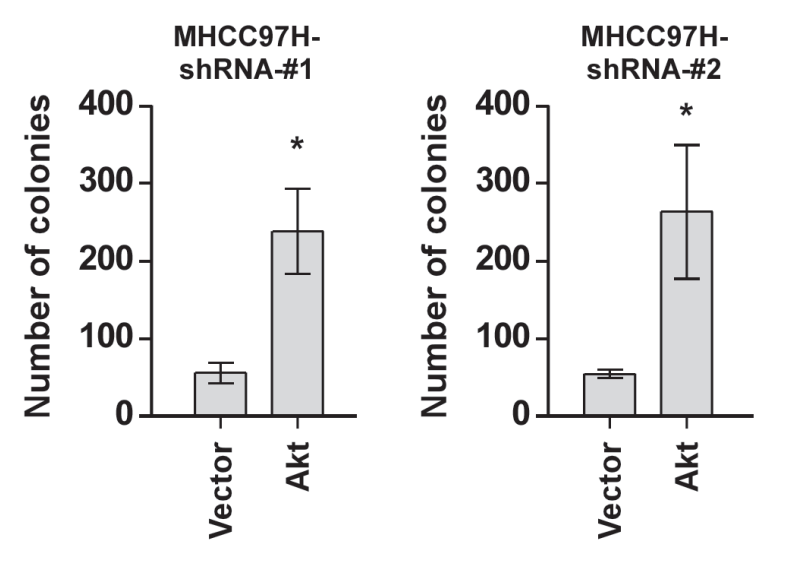

Figure 5. AKT has a crital role in EPS8L3 promotion of cell proliferation. A) The phosphoralytion level of AKT in Hep3B-EPS8L3 treated with AKT inhibitor, with $\alpha$-tubulin as the loading control. B) The colony numbers in EPS8L3-over-expressing cells effect on the AKT inhibitor. C) The colony numbers of EPS8L3-silencing cells from AKT participation. 
A

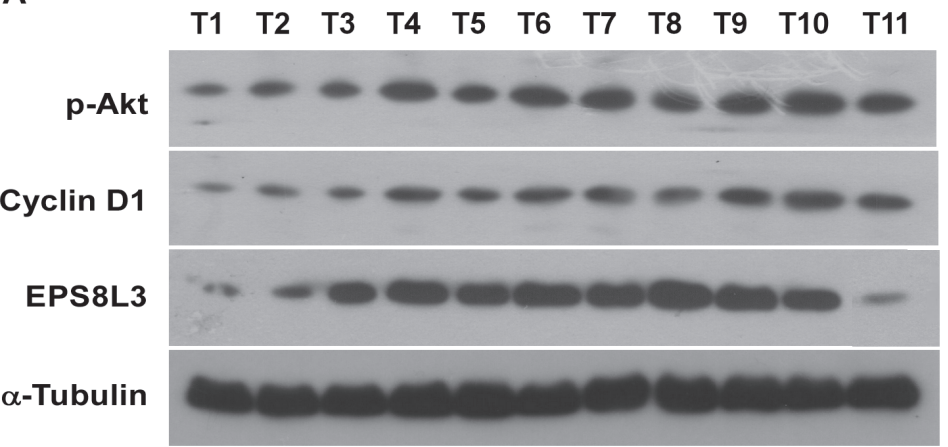

B
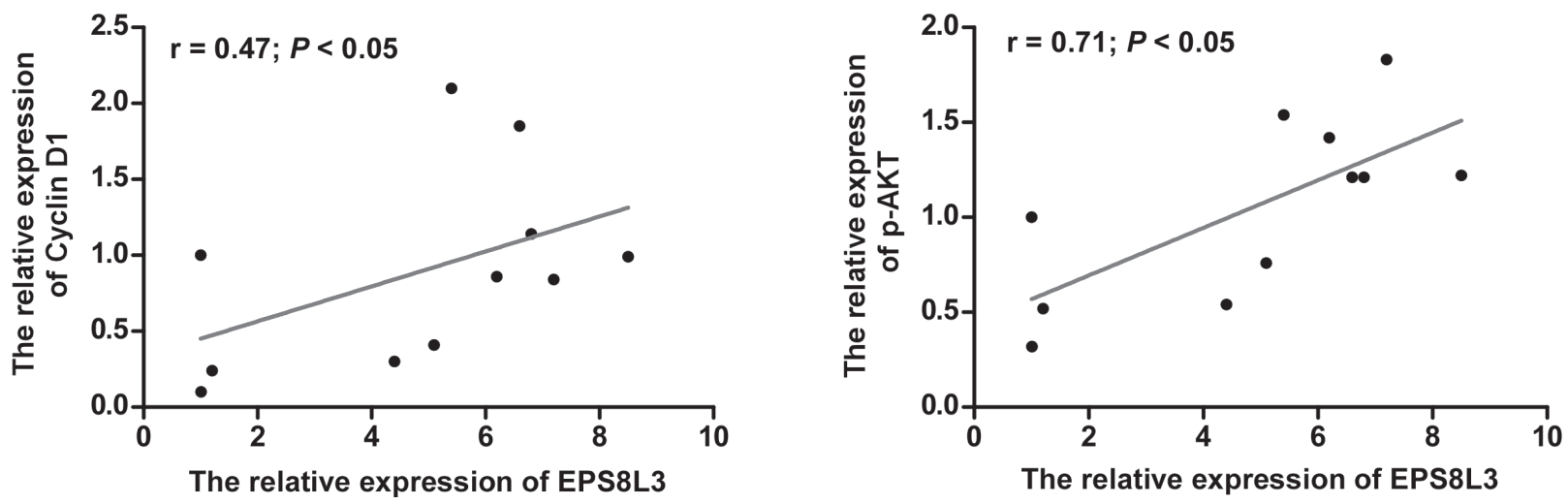

Figure 6. There is positive correlation between EPS8L3 and p-AKT or Cyclin D1 in 11 fresh HCC tissues. A) EPS8L3, p-AKT and Cyclin D1 protein levels in 11 HCC tissues, with $\alpha$-tubulin as the loading conrol. B) correlation analysis between EPS8L3 and Cyclin D1 (left panel; $r=0.35, p<0.05)$ and p-AKT (right panel; $\mathrm{r}=0.56, \mathrm{p}<0.05)$.

FRE-independent FOXO-binding elements in the Cyclin D1 promoter.

Although the precise inhibition mechanism remains unknown [22], this activity encourages anti-proliferation of cells. Our results highlight that EPS8L3 over-expression inhibits FOXO1 activity and increases Cyclin D1 expression and EPS8L3 therefore plays a vital role in HCC cell proliferation.

It is well documented that AKT regulates FOXO1 activity by phosphorylation $[23,24]$, and this supports our results; where $\mathrm{p}$-AKT and p-FOXO1 levels are both elevated in EPS8L3 over-expression of cells but inhibited in EPS8L3silenced cells. Therefore, EPS8L3 can regulate FOXO1 through AKT which is one of the major downstream genes of the epidermal growth factor receptor (EGFR). EGFR is ubiquitously expressed in human liver cells, and its signaling cascade has been recognized as pivotal in all stages of liver disease; from early inflammation and hepatocellular proliferation to fibrogenesis and neoplastic transformation [25, 26]. Further reports that EPS8 is a EGFR kinase substrate [9, 27] strengthen our hypothesis that EGFR regulates EPS8L3.
This then alters phosphorylation in the AKT and FOXO1 downstream gene, thus modulating cell proliferation. Proving this hypothesis is the intended object of our next contribution.

\section{References}

[1] TORRE LA1, BRAY F, SIEGEL RL, FERLAY J, LORTETTIEULENT J et al. Global cancer statistics, 2012. CA Cancer J Clin 2015; 65: 87-108. https://doi.org/10.3322/caac.21262

[2] CHEN W, ZHENG R, BAADE PD, ZHANG S, ZENG H et al. Cancer statistics in China, 2015. CA Cancer J Clin 2016; 66: 115-132. https://doi.org/10.3322/caac.21338

[3] SHIRVANI-DASTGERDI E, SCHWARTZ RE, PLOSS A. Hepatocarcinogenesis associated with hepatitis B, delta and C viruses. Curr Opin Virol. 2016; 20: 1-10. https://doi. org/10.1016/j.coviro.2016.07.009

[4] CASTAGNINO P, BIESOVA Z, WONG WT, FAZIOLI F, GILL GN et al. Direct binding of eps8 to the juxtamenbrane domain of EGFR is phosphotyrosine- and SH2-independent. Oncogene 1995; 10: 723-729. 
[5] LI YH, XUE TY, HE YZ, DU JW. Novel oncoprotein EPS8: a new target for anticancer therapy. Future Oncol 2013; 9: 1587-1594. https://doi.org/10.2217/fon.13.104

[6] DI FIORE PP, SCITA G. Eps8 in the midst of GTPases. Int J Biochem Cell Biol 2002; 34: 1178-1183.

[7] CHU PY, LIOU JH, LIN YM, CHEN CJ, CHEN MK et al. Expression of Eps8 correlates with poor survival in oral squamous cell carcinoma. Asia Pac J Clin Oncol 2012; 8: e77-81. https://doi.org/10.1111/j.1743-7563.2011.01459.x

[8] GORSIC LK, STARK AL, WHEELER HE, WONG SS, IM HK et al. EPS8 inhibition increase cisplatin sensitivity in lung cancer cells. PLoS One 2013; 8: e82220. https://doi. org/10.1371/journal.pone.0082220

[9] CHEN C, LIANG Z, HUANG W, LI X, ZHOU F et al. Eps8 regulates cellular proliferation and migration of breast cancer. Int J Oncol 2015; 46: 205-214. https://doi.org/10.3892/ ijo.2014.2710

[10] TOCCHETTI A, CONFALONIERI S, SCITA G, DI FIORE PP, BETSHOLTZ C. In silico analysis of the EPS8 gene family: genomic organization, expression profile, and protein structure. Genomics 2003; 81: 234-244.

[11] PRIETO-ECHAGUE V, CHAN PM, CRADDOCK BP, MANSER E, MILLER WT. PTB domain-directed substrate targeting in a tyrosine kinase from the unicellular choanoflagellate Monosiga brevicollis. PLoS One2011; 6: e19296. https://doi.org/10.1371/journal.pone.0019296

[12] Di Fiore PP, Scita G. EPS8 in the midst of GTPases. Int J Biochem Cell Biol 2002; 34: 1178-1183.

[13] TANG ED, NUNEZ G, BARR FG, GUAN KL. Negative regulation of the forkhead transcription factor FKHR by Akt. J Biol Chem 1999; 274: 16741-16746.

[14] ZENG Z, LIN H, ZHAO X, LIU G, WANG X et al. Overexpression of GOLPH3 promotes proligeration and tumorigenicity in breast cancer via suppression of the FOXO1 transcription factor. Clin Cancer Res 2012; 8: 4059-4069. https:// doi.org/10.1158/1078-0432.CCR-11-3156

[15] WANG X, LIN C, ZHAO X, LIU A, ZHU J et al. Acylglycerol kinase promotes cell proliferation and tumorigenicity in breast cancer via suppression of the FOXO1 transcription factor. Mol Cancer 2014; 13: 106. https://doi.org/10.1186/14764598-13-106

[16] KOPS GJ, MEDEMA RH, GLASSFORD J, ESSERS MA, DIJKERS PF et al. Control of cell cycle exit and entry by protein kinase B-regulated forkhead transcription factors. Mol Cell Biol 2002; 22: 2025-2036.

[17] WAGLE M, EIRING AM, WONGCHENKO M, LU S, GUAN Y et al. A role for FOXO1 in BCR-ABL1-independent tyrosine kinase inhibitor resistance in chronic myeloid leukemia. Leukemia 2016; 30: 1493-1501. https://doi. org/10.1038/leu.2016.51
[18] LLORETA J, FONT-TELLO A, JUANPERE N, FRANCES A, LORENZO $\mathrm{M}$ et al. FOXO1 down-regulation is associated with worse outcome in bladder cancer and adds significant prognostic information to p53 overexpression. Hum Pathol 2017; 62: 222-231. https://doi.org/10.1016/j.humpath.2016.12.022

[19] ZHANG Y, ZHANG L, SUN H, LV Q, QIU C et al. Forkhead transcription factor 1 inhibits endometrial cancer cell proliferation via sterol regulatory element-binding protein 1. Oncol Lett 2017; 13: 731-737. https://doi.org/10.3892/ ol.2016.5480

[20] LIU DZ, CHANG B, LI XD, ZHANG QH, ZOU YH. MicroRNA-9 promotes the proliferation, migration, and invasion of breast cancer via down-regulating FOXO1. Clin Transl Oncol 2017; 19: 1133-1140. https://doi.org/10.1007/ s12094-017-1650-1

[21] HUANG H, TINDALL DJ. Dynamic FoxO transcription factors. J Cell Sci 2007; 120: 2479-2487. https://doi. org/10.1242/jcs.001222

[22] SCHMIDT M, FERNANDEZ DE MATTOS S, VAN DER HORST A, KLOMPMAKER R, KOPS GJ et al. Cell cycle inhibition by FoxO forkhead transcription factors involves downregulation of cyclin D. Mol Cell Biol 2002; 22: 78427852 .

[23] ZHOU J, LIAO W, YANG J, MA K, LI X et al. FPXO3 induces FOXO1-dependent autophagy by activating the AKT1 signaling pathway. Autophagy 2012; 8: 1712-1723. https:// doi.org/10.4161/auto.21830

[24] LIANG R, RIMMELE P, BIGARELLA CL, YALCIN S, GHAFFARI S. Evidence for AKT-independent regulation of FOXO1 and FOXO3 in heematopoietic stem and progenitor cells. Cell Cycle 2016; 15: 861-867. https://doi.org/10.1080/1 5384101.2015.1123355

[25] BERASAIN C, AVILA MA. The EGFR signalling system in the liver: from hepatoprotection to hepatocarcinogenesis. J Gastroenterol 2014; 49: 9-23. https://doi.org/10.1007/ s00535-013-0907-x

[26] KOMPOSCH K, SIBILIA M. EGFR signaling in liver diseases. Int J Mol Sci 2015; 17: E30. https://doi.org/10.3390/ ijms 17010030

[27] FAZIOLI F, MINICHIELLO L, MATOSKA V, CASTAGNINO P, MIKI T et al. Eps8, a substrate for the epidermal growth factor receptor kinase, enhances EGF-dependent mitogenic signals. EMBO J 1993; 12: 3799-3808. 